\title{
Chronic and intermittent administration of systemic nitroglycerin in the rat induces an increase in the gene expression of CGRP in central areas: potential contribution to pain processing
}

Rosaria Greco $^{1 *}$ (D), Chiara Demartini ${ }^{1}$, Anna Maria Zanaboni ${ }^{1,2}$ and Cristina Tassorelli ${ }^{1,2}$

\begin{abstract}
Background: Calcitonin gene related peptide (CGRP) is a key neuropeptide involved in the activation of the trigeminovascular system and it is likely related to migraine chronification. Here, we investigated the role of CGRP in an animal model that mimics the chronic migraine condition via repeated and intermittent nitroglycerin (NTG) administration. We also evaluated the modulatory effect of topiramate on this experimental paradigm. Male SpragueDawley rats were injected with NTG (5 mg/kg, i.p.) or vehicle, every 2 days over a 9-day period (5 total injections). A group of animals was injected with topiramate (30 mg/kg, i.p.) or saline every day for 9 days. Twenty-four hours after the last administration of NTG or vehicle, animals underwent tail flick test and orofacial Von Frey test. Rats were subsequently sacrificed to evaluate c-Fos and CGRP gene expression in medulla-pons region, cervical spinal cord and trigeminal ganglia.
\end{abstract}

Results: NTG administration induced spinal hyperalgesia and orofacial allodynia, together with a significant increase in the expression of CGRP and c-Fos genes in trigeminal ganglia and central areas. Topiramate treatment prevented NTGinduced changes by reversing NTG-induced hyperalgesia and allodynia, and inhibiting CGRP and c-Fos gene expression in all areas evaluated.

Conclusions: These findings point to the role of CGRP in the processes underlying migraine chronification and suggest a possible interaction with gamma-aminobutyrate (GABA) and glutamate transmission to induce/maintain central sensitization and to contribute to the dysregulation of descending pain system involved in chronic migraine.

Keywords: Nitroglycerin, Topiramate, CGRP, Trigeminal nociception

\section{Background}

Pain persistence is associated with peripheral and central nervous system reorganization involving neuronal and glial changes [1]. Migraine chronification may result from maladaptive neuroplasticity along the nociceptive pathway [2]. Repeated trigeminal activation at the meningeal neurovascular endings indeed, with the associated neurogenic inflammation, may induce peripheral

\footnotetext{
* Correspondence: rosaria.greco@mondino.it

${ }^{1}$ Laboratory of Neurophysiology of Integrative Autonomic Systems, Headache Science Centre, IRCCS Mondino Foundation, Pavia, Italy Full list of author information is available at the end of the article
}

and central sensitization, which in turn predisposes patients to develop more migraine attacks in a vicious cycle that, in some migraineurs, leads to chronic migraine [3]. The mechanisms involved in migraine chronification are largely elusive; however, a major role seems to be played by the neuropeptide calcitonin gene-related peptide (CGRP), a vasodilatory peptide released by trigeminovascular endings to cause vasodilation, neurogenic inflammation and peripheral sensitization [4]. The role of CGRP in migraine attacks is reinforced by the efficacy of CGRP antagonism in animal models of migraine pain [5-7] and by promising reports on the efficacy of CGRP-related drugs 
in clinical trials, e.g. telcagepant or LY2951742 [8-10]. Recently, monoclonal antibodies directed against CGRP have proved effective in the preventive treatment of chronic migraine [11]. The precise mechanisms and sites where CGRP may act to favor chronification of migraine are still to be identified. CGRP receptors are largely distributed also in the brain, providing a wide range of possible CGRP targets and sites of interactions with other systems [12]. Topiramate is an antiepileptic drug with established efficacy in chronic migraine prevention $[13,14]$. The drug likely acts on the cell excitatory mechanisms via its influence on the receptor/channel protein complexes [15-17].

Nitroglycerin (NTG) is a nitric oxide (NO) donor that has been used for years as a provocative test in migraine for diagnostic and research purposes [18, 19]. In rodents, NTG induces an increased sensitivity to pain stimuli [20-22] and its chronic and intermittent administration causes acute plantar mechanical hyperalgesia with a progressive and sustained hyperalgesia [23, 24]. This behavior nicely reflects the increased sensitivity to painful stimuli in migraineurs [25] and therefore may be relevant for understanding the mechanism underlying migraine. Here, we aimed at gathering more insights into the role and mechanisms of CGRP in migraine chronification by chronic migraine-like rat model that mimics the condition of chronic migraine. In this model we evaluated: a) CGRP and c-Fos gene expression in areas involved in trigeminal nociception; b) the nociceptive threshold at the tail flick; c) orofacial mechanical allodynia; d) the modulatory effect of the migraine preventive drug topiramate.

\section{Methods}

\section{Animals}

We used adult male Sprague-Dawley rats (weight 200250 g, Charles River, s.r.l, Calco, Lecco, Italy) at the Centralized Animal Facility of the University of Pavia. The animals were housed under standard laboratory conditions in plastic boxes in groups of 2 with water and food available ad libitum and kept on a 12:12 h light-dark cycle, at room temperature of $19-21{ }^{\circ} \mathrm{C}$ with relative humidity of $70-80 \%$. All procedures were conducted in accordance with the European Convention for Care and Use of Laboratory Animals and with the IASP's guidelines for pain research in animals [26]. The experimental protocols were approved by the Italian Ministry of Health (Document number 1239/ 2015PR).

\section{Drugs}

Nitroglycerin (NTG) (Bioindustria L.I.M. Novi Ligure (AL), Italy) was prepared from a stock solution of $5.0 \mathrm{mg} / 1.5 \mathrm{~mL}$ dissolved in $27 \%$ alcohol and $73 \%$ propylene glycol. For the injections, NTG was further diluted in saline $(0.9 \% \mathrm{NaCl})$ to reach the final concentration of alcohol $6 \%$ and propylene glycol $16 \%$. The diluted NTG is injected intraperitoneally (i.p.) at the dose of $5 \mathrm{mg} / \mathrm{Kg}$ $[24,27]$. An equivalent volume of saline $(0.9 \% \mathrm{NaCl})$, alcohol $6 \%$ and propylene glycol 16\%) was used as vehicle. Topiramate (Topamax, Janssen-Cilag Cologno Monzese (MI), Italy) was dissolved in saline and administered i.p. at the dose of $30 \mathrm{mg} / \mathrm{Kg}$ [28]. Before baseline testing, rats were assigned to treatment groups according to a randomization list to ensure blinding to treatments of the researchers who performed the behavioral testing (tail flick test and Von Frey test, see below). Before testing the animals, the blinded examiners were instructed to observe the animal behavior, in order to evaluate the possible impact of topiramate on sedation or locomotor activities.

\section{Experimental design and experimental groups}

An a priori power analysis was conducted to determine the minimal sample size needed to obtain a statistical power of 0.80 at an alpha level of 0.05 . On the basis of our previous studies in rats evaluating the latency to the tail flick (sec.) before and after NTG, we calculated an effect size of 1.6 for this variable (GPower 3.1.9.2), estimating a sample size of at least 5 rats for experimental group. Rats were injected with NTG or vehicle, every 2 days over a 9-day total period (5 total injections). A group of animals was also injected with topiramate (TOP) or vehicle (saline) every day for 9 days. At baseline (day 0 ) and $24 \mathrm{~h}$ after the last administration of NTG (day 10), the animals belonging to the different experimental groups (see below) underwent the evaluation of the nociceptive threshold by means of the tail flick test and of the orofacial mechanical allodynia by means of the Von Frey test. The schedule of drug treatments is reported in Fig. 1.

Experimental Groups: CT $=$ NTG vehicle + TOP vehicle $(N=10-13$ per group); NTG $=$ NTG + TOP vehicle $(N=9-12$ per group $) ;$ TOP $=$ NTG vehicle + Topiramate $(N=5-8$ per group $) ; \mathrm{NTG}+\mathrm{TOP}=\mathrm{NTG}+$ Topiramate $(\mathrm{N}=5-8$ per group).

\section{Behavioral tests}

Rats underwent tail flick test and Von Frey test $30 \mathrm{~min}$ apart from each other at baseline and on day 10 .

\section{Tail flick test}

To determine thermal sensitivity, rats were gently restrained while an infrared light beam with a temperature of $50{ }^{\circ} \mathrm{C}$ was focused on the animal's tail. The latency of retraction of the tail from the window of the light beam was automatically captured and measured (in seconds) by means of a sensor (Ugo Basile, model 7360, Varese, Italy) [20, 21]. A cut-off limit of exposure of $20 \mathrm{~s}$ was set to avoid tissue damage. The final latency value for each animal was 


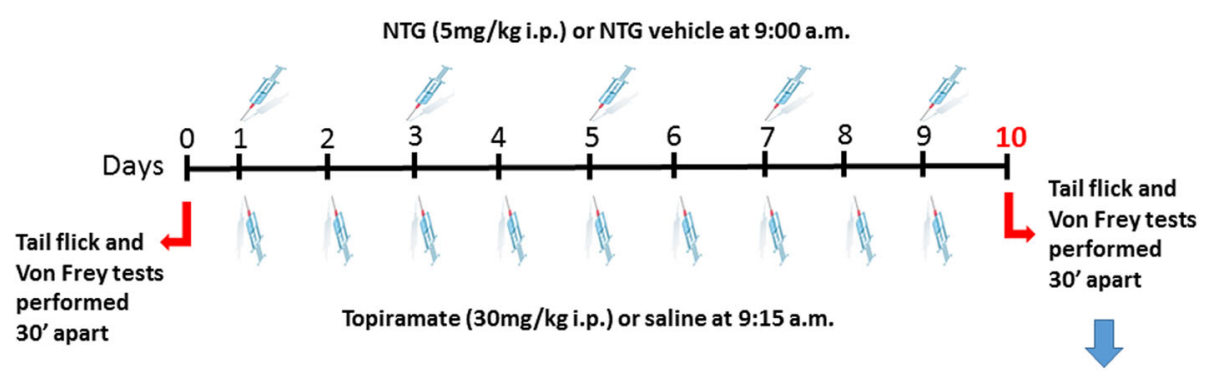

Sacrifice

Fig. 1 Experimental design and drugs administration schedule

calculated as the mean of four measurements in 4 different parts of the tail, specifically at 1, 3, 5 and $7 \mathrm{~cm}$ from the tip.

\section{Von Frey test}

For three consecutive days, prior to baseline testing, the rats were habituated to the behavioral test procedure. Rats were put in clear acrylic cages $(30 \times 30 \times 20 \mathrm{~cm})$ and the orofacial area of the animal was stimulated with a series of Von Frey filaments (bending force ranging from 0.02 to $6 \mathrm{~g})$. Progressively increasing filament forces were applied (each of them 5 times every $30 \mathrm{~s}$ ) to the cutaneous area on both the left and right sides of the face, over the rostral portion of the eye for periorbital testing and on the skin over the masseter muscle for jaw testing until we obtained a positive response, defined as one of the following: head withdrawal, face wipe, escape/attack. In the absence of a positive response to the starting filament $(0.4 \mathrm{~g})$, a heavier filament was applied whereas, with a positive response a lighter filament was tested. The mechanical threshold corresponded to the force of the filament that induced three positive responses $[29,30]$.

\section{CGRP and c-Fos gene expression}

On day 10, immediately after undergoing the Von Frey test, all rats were sacrificed and their medulla-pons region, cervical spinal cord (C1-C2) and trigeminal ganglia were immediately chopped into parts for the evaluation of CGRP and c-Fos gene expression. mRNA levels were analyzed by real-time polymerase chain reaction (RT-PCR) as previously described [22, 31]. Primer sequences of Calca gene, coding for CGRP, (forward primer: CAGT CTCAGCTCCAAGTCATC; reverse primer: TTCC AAGGTTGACCTCAAAG) and c-Fos (forward primer: TACGCTCCAAGCGGAGAC; reverse primer: TTTCCTTCTCTTTCAGTAGATTGG) were obtained from the AutoPrime software (http://www.autoprime.de/ AutoPrimeWeb). We used glyceraldehyde 3-phosphate dehydrogenase (GAPDH; forward primer: AACCTGCCA AGTATGATGAC; reverse primer: GGAGTTGCTGTTGA AGTCA) as housekeeping gene. All samples were assayed in triplicate. Gene expression was calculated using the $\Delta \Delta \mathrm{Ct}$ method.

\section{Data analysis and statistical evaluation}

Our data showed a normal distribution when analyzed with the Kolmogorov-Smirnov (KS) normality tests. For the behavioral tests the inter-groups and within groups differences between baseline and post-treatment timings were analyzed using the Two-way ANOVA for repeated measures followed by Bonferroni post hoc test. For gene expression, the differences between groups were analyzed by One-way ANOVA followed by Newman-Keuls multiple comparison test. A $p$ value $<0.05$ was considered statistically significant. The results were reported in the figures and all data were expressed as the mean + standard error of the mean. The Statistical analysis was performed using GraphPad Prism 5.02.

\section{Results}

\section{Behavioral testing}

No distinctive pattern of behavior was identified in the different experimental groups by comparing the reports of the blinded observers after treatment unblinding.

\section{Tail Flick test}

No significant difference among CT, NTG and NTG + TOP groups was observed at baseline (Fig. 2a). Chronic NTG administration caused a state of hyperalgesia, which was detected as a reduction in the latency at the tail flick test performed on day 10 as compared with both baseline value and CT group. The chronic administration of topiramate did not influence the tail flick latency, but it prevented NTG-induced hyperalgesic state. Data are reported in Fig. 2a.

\section{Von Frey test}

We did not detect any significant difference in baseline mechanical sensitivity among experimental groups (Fig. 2b). Chronic and intermittent NTG administration caused orofacial allodynia with a reduction in the mechanical pain threshold compared to the baseline value and to 

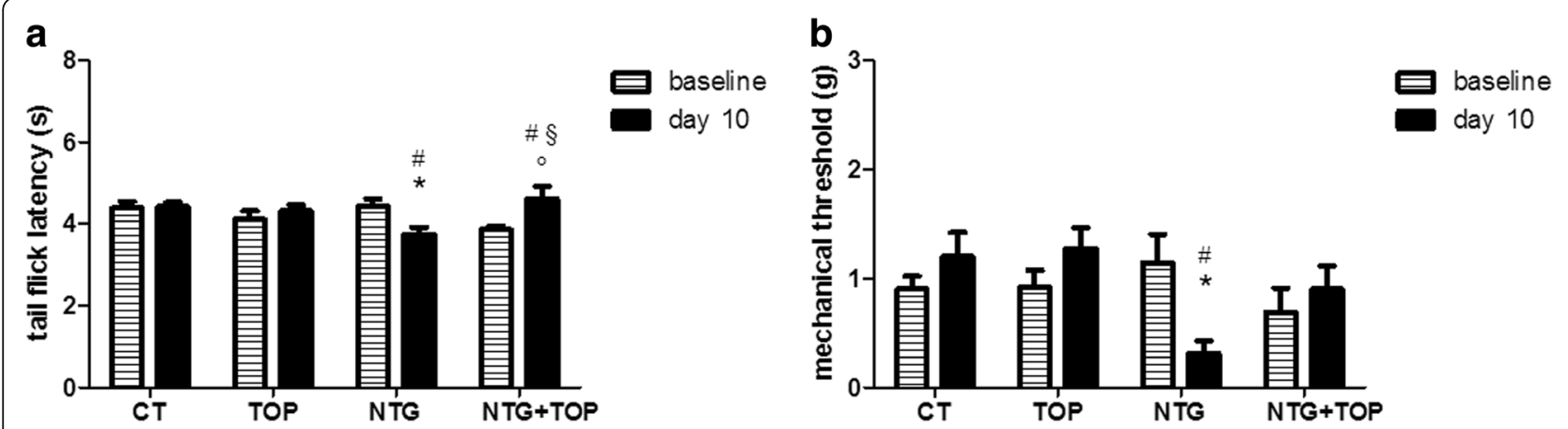

Fig. $\mathbf{2}$ a Nociceptive thermal threshold (tail flick test) and $\mathbf{b}$ orofacial mechanical allodynia (Von Frey test) following chronic and intermittent NTG administration and chronic topiramate (TOP) treatment in rats. CT $=$ NTG vehicle + TOP vehicle $(n=13)$; NTG $=$ NTG + TOP vehicle $(n=12) ;$ TOP $=$ NTG vehicle + Topiramate $(n=8) ;$ NTG + TOP $=$ NTG + Topiramate $(n=8)$. Data are expressed as mean + SEM; two-way ANOVA followed by Bonferroni post hoc tests, $F=6.019$ for tail flick test; $F=4.091$ for Von Frey test. ${ }^{*} p<0.05$ vs NTG baseline; ${ }^{\circ} p<0.05$ vs NTG + TOP baseline; \#p $<0.05$ vs CT and TOP 1 day 10; $\$ p<0.05$ vs NTG day 10

the CT group. Topiramate did not change the mechanical pain threshold when compared to the baseline value and to the CT group, but it prevented the development of NTG-induced allodynia. The mechanical threshold of NTG + TOP was increased compared to NTG group, although it did not reach a statistical significant level, probably because of the great variability of data. Data are reported in Fig. 2b.
mRNA expression of CGRP and c-Fos genes

NTG administration induced a significant increase in the expression of c-Fos and CGRP mRNA in the medulla-pons region, cervical spinal cord and trigeminal ganglia (Fig. 3). Topiramate did not modify genes expression in these areas when used alone, but it markedly attenuated CGRP and c-Fos gene expression induced by NTG in all the areas under evaluation (Fig. 3).

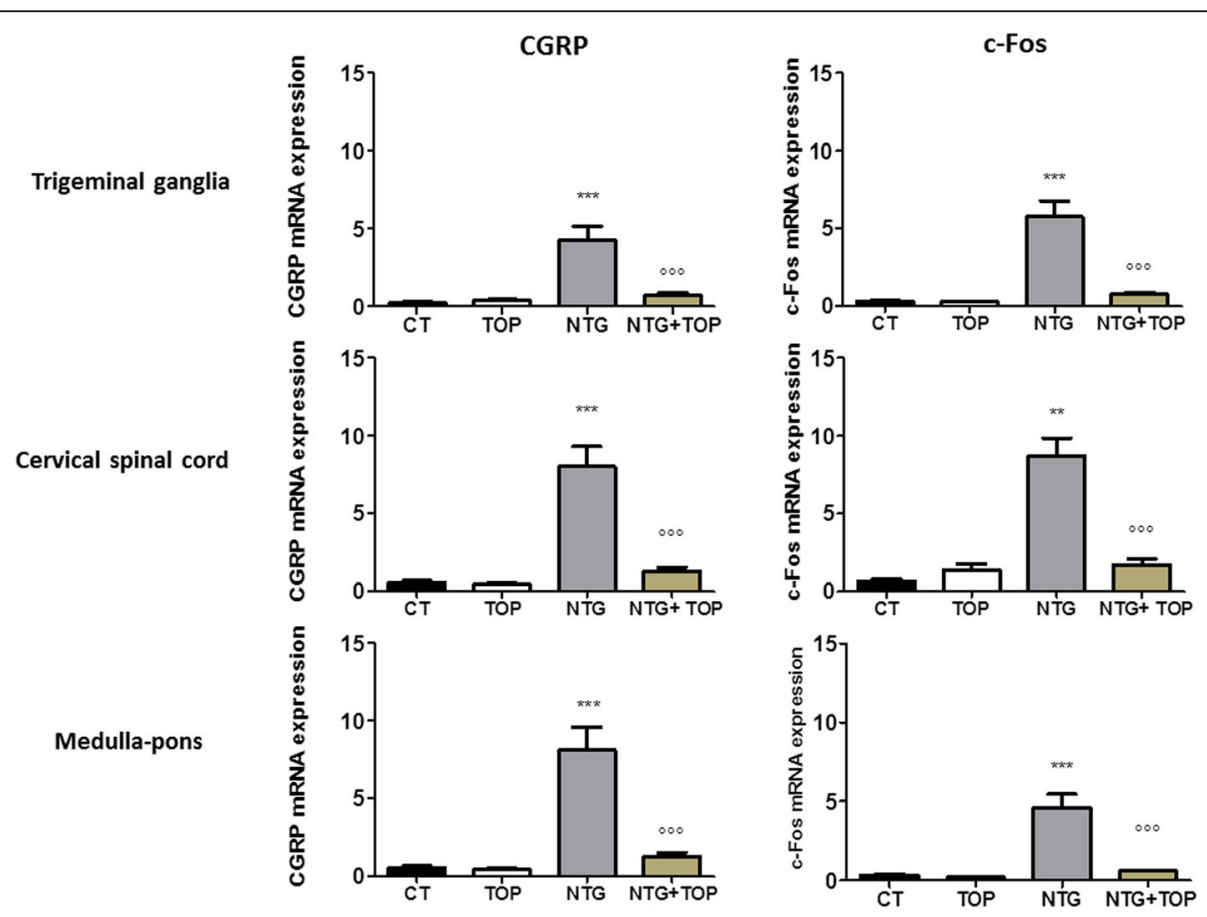

Fig. 3 CGRP and c-Fos mRNA expression in areas involved in trigeminal nociception, following chronic and intermittent NTG administration in rat. $\mathrm{CT}=\mathrm{NTG}$ vehicle $+\mathrm{TOP}$ vehicle $(n=10) ; \mathrm{NTG}=\mathrm{NTG}+\mathrm{TOP}$ vehicle $(n=9) ; \mathrm{TOP}=\mathrm{NTG}$ vehicle + Topiramate $(n=5) ; \mathrm{NTG}+\mathrm{TOP}=\mathrm{NTG}+$ Topiramate $(n=5)$. Data are expressed as mean + SEM. One way ANOVA followed by Newman-Keuls Multiple Comparison Test ${ }^{* *} p<0.01$ and ${ }^{* * *} p<0.001$ vs $C T$ and TOP; ${ }^{000} p<0.001$ vs NTG 


\section{Discussion}

Migraine is a highly disabling condition [32] that manifests with recurring attacks. In $2-3 \%$ of the general population it becomes chronic ( $\geq 15$ days/months) and therefore even more disabling. Progressive changes in nociceptive thresholds and subsequent central sensitization due to recurrent migraine headaches in vulnerable subjects contribute to the chronic migraine state. Understanding the mechanisms of chronification seems therefore extremely useful. The role of CGRP in the precipitation of migraine attacks is nowadays well accepted [33]. Less known is CGRP role in the processes that lead to migraine chronification.

Several studies indicate that NTG administration in rodents is a predictive model of migraine in humans $[24,34,35]$. In this study we aimed at developing and testing a chronic migraine-like model in rats, based on chronic and intermittent administration of NTG. In this model the animals become hyperalgesic and allodynic, which nicely mimics the clinical picture on one side [25, 36-39], and, on the other, parallels the findings obtained by Pradhan et al. in the animal model of chronic migraine devised in mice [24]. The availability of an animal model of chronic migraine in rats provides a precious opportunity for investigating the pathophysiological mechanisms involved in chronic migraine for a dual reason: 1) rat models have technical and practical advantages [40] and 2) the rat-based migraine model of acute NTG administration is well-established and has already yielded a wealth of data from several groups [41, 42].

Using this chronic migraine-like model we showed that the observed changes in pain perception - namely a reduction in the latency of the tail flick test and orofacial mechanical allodynia - are paralleled by an increased expression in CGRP mRNA in the trigeminal ganglia. CGRP is involved in the maintenance of ongoing sensitization and the increased gene expression observed in our model may be interpreted as a compensatory mechanism for reintegrating CGRP released at the trigeminovascular endings [43-45]. In agreement, Farajdokht et al. [46] demonstrated that the increase in CGRP gene expression in trigeminal ganglia is associated with increased transient receptor potential vanilloid type-1 mRNA levels in the same area $48 \mathrm{~h}$ after NTG injection. Previous reports have suggested that NTG induces hyperalgesia via complex mechanisms that involve also sensitization of the trigeminovascular system at the meningeal level [47-49]. Previously, we reported that acute NTG administration did not cause any change in CGRP-immunoreactivity in the lumbar dorsal horns, whereas it increased SP staining intensity in both the cervical and lumbar spinal cord $1 \mathrm{~h}$ after NTG administration [43]. Thus, though the effect of NTG administration seems relevant for processes that take place in the cervical spinal cord, we cannot rule out the occurrence of systemic NTG effects on other neuronal subpopulations and other areas of the nervous system. Of note, in a previous study Edelmeyer et al. [50] showed that inflammatory mediators applied to the dura of non-anesthetized rats caused strong and time-related facial and hindpaw allodynia, thus suggesting that the alterations in pain sensitivity associated with primary headache, probably reflect a more generalized condition of central sensitization [50].

In a previous study, we showed that acute NTG administration induces an increase in CGRP gene expression in the trigeminal ganglia, cervical spinal cord and medulla-pons area $5 \mathrm{~h}$ after the administration $[31,51]$. Here, we show that the levels of CGRP mRNA are elevated in the same areas $24 \mathrm{~h}$ after the last dose of NTG administration. These findings are consistent with the synthesis and release of CGRP in these central areas, where the peptide can modulate nociceptive transmission [52]. Indeed, CGRP mRNA signal has been detected in the nucleus trigeminal caudalis (NTC) and other brain structures of naïve rats [53, 54], which include the nucleus tractus solitarius, receiving inhibitory baroreceptor afferents, the ventrolateral medulla, a key structure in the descending pain modulation, and the pontine parabrachial nucleus $(\mathrm{PBN})$ that contributes to nociceptive transmission with its projections to the central nucleus of the amygdala (CeA) [55]. More importantly, CGRP and its receptor antagonists act on neurons in the ventrolateral periaqueductal gray to influence nociceptive transmission in the nucleus tractus caudalis. Functional CGRP receptors are present as well in other regions of the brain that are involved in the modulation of migraine pain [56]. Taken together, these observations support the contribution of CGRP in central sensitization and in the functioning of descending pain pathways, both of which are implicated in chronic migraine [57].

To further test the relevance of our animal model of chronic migraine we evaluated the effect of chronic administration of topiramate, one of the few drugs that have proved effective in the preventive treatment of chronic migraine in randomized controlled trials $[58,59]$.

Topiramate reduces brain hyperexcitability, a hallmark of migraine [60], via multiple mechanisms: blockade of voltage dependent sodium channels, increased activity of the neurotransmitter gamma-aminobutyrate (GABA) and antagonism of the $\alpha$-amino-3-hydroxy5-methyl-4-isoxazolepropionic acid (AMPA)/kainate subtype of the glutamate receptor and inhibition of the carbonic anhydrase enzyme [61, 62]. An in vitro study demonstrated that topiramate is able to attenuate neurogenic dural vasodilation by inhibiting the release of CGRP from prejunctional trigeminal neurons, thus suggesting an interaction with this neuropeptide [15].

Chronic treatment with topiramate in our model reduced trigeminal spinal hyperalgesia and mechanical allodynia, while diminishing CGRP mRNA levels in the areas under investigation. This finding seems in agreement with 
preclinical studies showing that topiramate has an anti-allodynic effect in neuropathic pain at doses of 20$50 \mathrm{mg} / \mathrm{kg}[28,63-65]$. Chronic administration of topiramate $(50 \mathrm{mg} / \mathrm{kg} /$ day, i.p.) to neuropathic rats diminished the mechanical sensitivity and shortened the period of allodynia to 8 days [28].

CGRP can directly activate the nociceptors or facilitate their firing, thus causing peripheral sensitization and hyperalgesia [1]. In this frame, it is possible that the anti-hyperalgesic effect of topiramate observed in the present study is related to the inhibitory activity on the neuronal firing within the trigeminocervical complex and the ventroposteromedial thalamic nucleus, as reported by Andreou and Goadsby [66].

Topiaramate blocks voltage-dependent sodium channel in spinal cord neurons, which prompts a possible mechanism for reducing afferent input to the NTC [67]. Other mechanisms could be involved such as anti-inflammatory and immunomodulatory effects or inhibition of neurotransmitters release $[68,69]$. Central sensitization involves an increased sensitivity of second-order neurons to afferent inputs, the augmentation of receptive fields and an increased excitability; furthermore, it involves long-term effects related to transcriptional changes and dysfunctions in the descending pain system [70]. The maintenance of central sensitization may be related to the activation of the glutamate N-methyl-D-aspartate (NMDA), AMPA and metabotropic receptors. Thus, AMPA/kainate receptors inhibition by topiramate treatment could decrease neuronal hyperexcitability within the NTC and brain structures receiving inputs from second order neurons [68]. Glutamate-like immunoreactivity has been indeed detected in tooth pulp neurons that project to the NTC in the rat [71].

It is worth noting that in our experimental paradigm, topiramate treatment significantly reduced CGRP gene expression in cervical spinal cord and medulla-pons. In agreement, topiramate treatment repressed $\mathrm{KCl}$ stimulated CGRP release in a time and concentration dependent manner in trigeminal cultures [72]. However, since GABAergic mechanisms have been shown to be implicated in the trigeminocervical complex, it is stimulating to hypothesize that GABA potentiation due to topiramate treatment may interfere with CGRP synthesis in the NTC, as well as in the areas involved in the descending pain system [34, 35, 57, 73]. Synaptic contacts between CGRP-positive terminals and GABAergic neurons were found within the CeA, which may underlie the pain-related neural pathway from PBN to $\mathrm{CeA}$, in chronic pain modulation.

The increased c-Fos mRNA levels found $24 \mathrm{~h}$ after NTG treatment, confirms that NTG-induced transcriptional changes are not limited to peripheral areas, but rather affect central structures, directly or indirectly, thus reflecting a widespread, peripheral and central diffusion of sensitization phenomena consistent with the observed alterations in pain responses. While a large body of information has been collected over the years by our group and others on the acute NTG model, the mechanisms responsible for c-Fos gene expression after chronic NTG are almost completely unknown. At this time, we can only speculate that the model proposed in this study actually engages the CNS with persisting functional and morphological changes associated to chronic sensitization.

\section{Limitations of the study}

The design of study foresaw the chronic administration of topiramate. Data from the literature suggest that acute administration of topiramate reduces neuronal firing in the trigeminovascular complex [66] therefore the chronic administration used in our design might not have been necessary. In the absence of reliable evidence, we selected the chronic administration for several reasons. First, this treatment schedule reflects more closely the clinical scenario, where topiramate is used daily over months to prevent migraine attacks. Second, data is lacking on the serum/plasma concentration of topiramate in the rat. Human studies show that the plasma half-life of the drug is $20-30 \mathrm{~h}$ after a single oral administration [74, 75], while it is shorter in rats [76], thus posing the issue of unstable blood levels of topiramate. This could have been addressed by increasing the dose of the single administration, but also the possibility that the sedative effect of such a higher dose might interfere with the nocifensive behavior of the rat [77]. The lack of precise pharmacokinetics studies on repeated dosing in the laboratory animal ultimately suggested the selection of dose regimens based on a best-guess modality, guided by careful evaluation of available evidence.

Future studies are needed to address topiramate serum/ plasma concentrations in our model, together with the evaluation of the potential impact of topiramate-induced locomotor activity in the nocifensive behavior of chronically NTG-treated rats using specific behavioral testing.

\section{Conclusions}

The present findings, obtained in a novel chronic migrainelike model in the rat, suggest that CGRP pathway activation is involved in the facilitation of nociceptive transmission and is likely to contribute to central sensitization and dysfunction in descending pain control, via modulatory effects that encompass the modulation of voltage dependent sodium channels, GABA activity and the glutamate pathway. A fuller understanding of CGRP effects and CGRP network in brain regions will provide powerful insights for understanding the complex circuitry of migraine and for the improvement of migraine therapies.

\section{Abbreviations}

AMPA: $a$-amino-3-hydroxy-5-methyl-4-isoxazolepropionic acid; CeA: Central nucleus of the amygdala; CGRP: Calcitonin gene-related peptide; 
GABA: Gamma-aminobutyrate; GAPDH: Glyceraldehyde 3-phosphate dehydrogenase; NMDA: N-methyl-D-aspartate; NO: Nitric oxide; NTC: trigeminal nucleus caudalis; NTG: Nitroglycerin; PBN: Pontine parabrachial nucleus; RTPCR: Real-time polymerase chain reaction; TOP: Topiramate

\section{Funding}

This work was supported by a grant of the Italian Ministry of Health (Ricerca Corrente 2016) to the IRCCS Mondino Foundation.

\section{Authors' contributions}

$R G$ designed the experiments and drafted the manuscript. $C D$ and $A Z$ performed the experiments. RG analyzed and interpreted the data. CT revised the manuscript. All authors read and approved the final manuscript.

\section{Ethics approval and consent to participate}

All procedures were in accordance with the European Convention for Care and Use of Laboratory Animals and were approved by the Italian Ministry of Health (Document number 1239/2015PR).

\section{Consent for publication}

Not applicable.

\section{Competing interests}

The authors declare that they have no competing interests.

\section{Publisher's Note}

Springer Nature remains neutral with regard to jurisdictional claims in published maps and institutional affiliations.

\section{Author details}

${ }^{1}$ Laboratory of Neurophysiology of Integrative Autonomic Systems, Headache Science Centre, IRCCS Mondino Foundation, Pavia, Italy. ${ }^{2}$ Department of Brain and Behavioral Sciences, University of Pavia, Pavia, Italy.

Received: 13 March 2018 Accepted: 2 July 2018

Published online: 13 July 2018

\section{References}

1. Basbaum Al, Bautista DM, Scherrer G, Julius D (2009) Cellular and molecular mechanisms of pain. Cell 139(2):267-284

2. Latremoliere A, Woolf CJ (2009) Central sensitization: a generator of pain hypersensitivity by central neural plasticity. J Pain 10(9):895-926

3. Bernstein C, Burstein R (2012) Sensitization of the trigeminovascular pathway: perspective and implications to migraine pathophysiology. J Clin Neurol 8(2):89-99

4. lyengar S, Ossipov MH, Johnson KW (2017) The role of calcitonin generelated peptide in peripheral and central pain mechanisms including migraine. Pain 158(4):543-559

5. Greco R, Mangione AS, Siani F, Blandini F, Vairetti M, Nappi G, Sandrini G, Buzzi MG, Tassorelli C (2014) Effects of CGRP receptor antagonism in nitroglycerin-induced hyperalgesia. Cephalalgia 34(8):594-604

6. Cornelison LE, Hawkins JL, Durham PL (2016) Elevated levels of calcitonin gene-related peptide in upper spinal cord promotes sensitization of primary trigeminal nociceptive neurons. Neuroscience 339:491-501

7. Filiz A, Tepe N, Eftekhari S, Boran HE, Dilekoz E, Edvinsson L, Bolay H (2017) CGRP receptor antagonist MK-8825 attenuates cortical spreading depression induced pain behavior. Cephalalgia. https://doi.org/10.1177/ 0333102417735845

8. Ho TW, Connor KM, Zhang Y, Pearlman E, Koppenhaver J, Fan X, Lines C, Edvinsson L, Goadsby PJ, Michelson D (2014) Randomized controlled trial of the CGRP receptor antagonist telcagepant for migraine prevention. Neurology 83(11):958-966

9. Ho TW, Ho AP, Ge YJ, Assaid C, Gottwald R, MacGregor EA, Mannix LK, van Oosterhout WP, Koppenhaver J, Lines C, Ferrari MD, Michelson D (2016) Randomized controlled trial of the CGRP receptor antagonist telcagepant for prevention of headache in women with perimenstrual migraine. Cephalalgia 36(2):148-161

10. Dodick DW, Goadsby PJ, Spierings EL, Scherer JC, Sweeney SP, Grayzel DS (2014) Safety and efficacy of LY2951742, a monoclonal antibody to calcitonin gene-related peptide, for the prevention of migraine: a phase 2, randomised, double-blind, placebo-controlled study. Lancet Neurol 13(9):885-892

11. Silberstein SD, Dodick DW, Bigal ME, Yeung PP, Goadsby PJ, Blankenbiller T, Grozinski-Wolff M, Yang R, Ma Y, Aycardi E (2017) Fremanezumab for the preventive treatment of chronic migraine. N Engl J Med 377(22): 2113-2122

12. Russo AF (2015) Calcitonin gene-related peptide (CGRP): a new target for migraine. Annu Rev Pharmacol Toxicol 55:533-552

13. Brandes JL, Saper JR, Diamond M, Couch JR, Lewis DW, Schmitt J, Neto W, Schwabe S, Jacobs D, MIGR-002 Study Group (2004) Topiramate for migraine prevention: a randomized controlled trial. JAMA 291(8):965-973

14. Silberstein SD (2017) Topiramate in migraine prevention: a 2016 perspective. Headache 57(1):165-178

15. Akerman S, Goadsby PJ (2005) Topiramate inhibits trigeminovascular activation: an intravital microscopy study. Br J Pharmacol 146(1):7-14

16. Motaghinejad M, Motevalian M (2016) Involvement of AMPA/kainate and GABAA receptors in topiramate neuroprotective effects against methylphenidate abuse sequels involving oxidative stress and inflammation in rat isolated hippocampus. Eur J Pharmacol 784:181-191

17. Curia G, Aracri P, Colombo E, Scalmani P, Mantegazza M, Avanzini G, Franceschetti S (2007) Phosphorylation of sodium channels mediated by protein kinase-C modulates inhibition by topiramate of tetrodotoxinsensitive transient sodium current. Br J Pharmacol 150(6):792-797

18. Sances G, Tassorelli C, Pucci E, Ghiotto N, Sandrini G, Nappi G (2004) Reliability of the nitroglycerin provocative test in the diagnosis of neurovascular headaches. Cephalalgia 24(2):110-119

19. Ashina M, Hansen JM, Olesen J (2013) Pearls and pitfalls in human pharmacological models of migraine: 30 years' experience. Cephalalgia 33(8):540-553

20. Greco R, Tassorelli C, Armentero MT, Sandrini G, Nappi G, Blandini F (2008) Role of central dopaminergic circuitry in pain processing and nitroglycerininduced hyperalgesia. Brain Res 1238:215-223

21. Greco R, Bandiera T, Mangione AS, Demartini C, Siani F, Nappi G, Sandrini G, Guijarro A, Armirotti A, Piomelli D, Tassorelli C (2015) Effects of peripheral FAAH blockade on NTG-induced hyperalgesia-evaluation of URB937 in an animal model of migraine. Cephalalgia 35(12):1065-1076

22. Greco R, Siani F, Demartini C, Zanaboni A, Nappi G, Davinelli S, Scapagnini G, Tassorelli C (2016) Andrographis Paniculata shows anti-nociceptive effects in an animal model of sensory hypersensitivity associated with migraine. Funct Neurol 31(1):53-60

23. Farajdokht F, Babri S, Karimi P, Alipour MR, Bughchechi R, Mohaddes G (2017) Chronic ghrelin treatment reduced photophobia and anxiety-like behaviors in nitroglycerin- induced migraine: role of pituitary adenylate cyclase-activating polypeptide. Eur J Neurosci 45(6):763-772

24. Pradhan AA, Smith ML, McGuire B, Tarash I, Evans CJ, Charles A (2014) Characterization of a novel model of chronic migraine. Pain 155(2):269-274

25. Perrotta A, Serrao M, Tassorelli C, Arce-Leal N, Guaschino E, Sances G, Rossi P, Bartolo M, Pierelli F, Sandrini G, Nappi G (2011) Oral nitric-oxide donor glyceryl-trinitrate induces sensitization in spinal cord pain processing in migraineurs: a double-blind, placebo-controlled, cross-over study. Eur J Pain 15(5):482-490

26. Zimmerman M (1983) Ethical guidelines for investigations of experimental pain in conscious animals. Pain 16:109-110

27. Tassorelli C, Greco R, Cappelletti D, Sandrini G, Nappi G (2005) Comparative analysis of the neuronal activation and cardiovascular effects of nitroglycerin, sodium nitroprusside and L-arginine. Brain Res 1051(1-2):17-24

28. Wieczorkiewicz-Płaza A, Płaza P, Maciejewski R, Czuczwar M, Przesmycki K (2004) Effect of topiramate on mechanical allodynia in neuropathic pain model in rats. Pol J Pharmacol 56(2):275-278

29. Martin YB, Avendaño C (2009) Effects of removal of dietary polyunsaturated fatty acids on plasma extravasation and mechanical allodynia in a trigeminal neuropathic pain model. Mol Pain 5:8

30. Oshinsky ML, Sanghvi MM, Maxwell CR, Gonzalez D, Spangenberg RJ, Cooper M, Silberstein SD (2012) Spontaneous trigeminal allodynia in rats: a model of primary headache. Headache 52(9):1336-1349

31. Demartini C, Tassorelli C, Zanaboni AM, Tonsi G, Francesconi O, Nativi C, Greco R (2017) The role of the transient receptor potential ankyrin type-1 (TRPA1) channel in migraine pain: evaluation in an animal model. J Headache Pain 18(1):94

32. GBD 2015 Neurological Disorders Collaborator Group (2017) Global, regional, and national burden of neurological disorders during 1990-2015: a 
systematic analysis for the Global Burden of Disease Study 2015. Lancet Neurol 16(11):877-897

33. Bigal ME, Walter S, Rapoport AM (2013) Calcitonin gene-related peptide (CGRP) and migraine current understanding and state of development. Headache 53(8):1230-1244

34. Moye LS, Pradhan AAA (2017) Animal model of chronic migraine-associated pain. Curr Protoc Neurosci 80:9.60.1-9.60.9

35. Farkas S, Bölcskei K, Markovics A, Varga A, Kis-Varga Á, Kormos V, Gaszner B, Horváth C, Tuka B, Tajti J, Helyes Z (2016) Utility of different outcome measures for the nitroglycerin model of migraine in mice. J Pharmacol Toxicol Methods 77:33-44

36. de Tommaso M, Ambrosini A, Brighina F, Coppola G, Perrotta A, Pierelli F, Sandrini G, Valeriani M, Marinazzo D, Stramaglia S, Schoenen $J(2014)$ Altered processing of sensory stimuli in patients with migraine. Nat Rev Neurol 10(3):144-155

37. Sandrini G, Tassorelli C, Cecchini AP, Alfonsi E, Nappi G (2002) Effects of nimesulide on nitric oxide-induced hyperalgesia in humans-a neurophysiological study. Eur J Pharmacol 450(3):259-262

38. Burstein R, Jakubowski M, Rauch SD (2011) The science of migraine. J Vestib Res 21(6):305-314

39. Louter MA, Bosker JE, van Oosterhout WP, van Zwet EW, Zitman FG, Ferrari MD, Terwindt GM (2013) Cutaneous allodynia as a predictor of migraine chronification. Brain 136(Pt 11):3489-3496

40. Ellenbroek B, Youn J (2016) Rodent models in neuroscience research: is it a rat race? Dis Model Mech 9(10):1079-1087

41. Valença MM (2017) Commentary: the effect of systemic nitroglycerin administration on the kynurenine pathway in the rat. Front Neurol 8:518

42. Ramachandran R, Pedersen SH, Amrutkar DV, Petersen S, Jacobsen JM, HaySchmidt A, Olesen J, Jansen-Olesen I (2017) Selective cephalic upregulation of $\mathrm{p}$-ERK, CamKII and p-CREB in response to glyceryl trinitrate infusion. Cephalalgia Jan 1:333102417722511

43. Greco R, Tassorelli C, Sandrini G, Di Bella P, Buscone S, Nappi G (2008) Role of calcitonin gene-related peptide and substance $P$ in different models of pain. Cephalalgia 28(2):114-126

44. Pardutz A, Multon S, Malgrange B, Parducz A, Vecsei L, Schoenen J (2002) Effect of systemic nitroglycerin on CGRP and 5 -HT afferents to rat caudal spinal trigeminal nucleus and its modulation by estrogen. Eur J Neurosci 15(11):1803-1809

45. Capuano A, Greco MC, Navarra P, Tringali G (2014) Correlation between algogenic effects of calcitonin-gene-related peptide (CGRP) and activation of trigeminal vascular system, in an in vivo experimental model of nitroglycerin-induced sensitization. Eur J Pharmacol 740:97-102

46. Farajdokht F, Mohaddes G, Shanehbandi D, Karimi P, Babri S (2017) Ghrelin attenuated hyperalgesia induced by chronic nitroglycerin: CGRP and TRPVI as targets for migraine management. Cephalalgia. Jan 1:333102417748563. https://doi.org/10.1177/0333102417748563

47. Tassorelli C, Joseph SA, Buzzi MG, Nappi G (1999) The effects on the central nervous system of nitroglycerin-putative mechanisms and mediators. Prog Neurobiol 57(6):607-624

48. Buzzi MG, Tassorelli C (2010) Experimental models of migraine. Handb Clin Neurol 97:109-123

49. Reuter U, Bolay H, Jansen-Olesen I, Chiarugi A, Sanchez del Rio M Letourneau R, Theoharides TC, Waeber C, Moskowitz MA (2001) Delayed inflammation in rat meninges: implications for migraine pathophysiology. Brain 124(Pt 12):2490-2502

50. Edelmayer RM, Ossipov MH, Porreca F (2012) An experimental model of headache-related pain. Methods Mol Biol 851:109-120

51. Greco R, Demartini C, Zanaboni AM, Redavide E, Pampalone S, Toldi J, Fülöp F, Blandini F, Nappi G, Sandrini G, Vécsei L, Tassorelli C (2017) Effects of kynurenic acid analogue 1 (KYNA-A1) in nitroglycerininduced hyperalgesia: targets and anti-migraine mechanisms. Cephalalgia 37(13):1272-1284

52. Raddant AC, Russo AF (2011) Calcitonin gene-related peptide in migraine: intersection of peripheral inflammation and central modulation. Expert Rev Mol Med 13:e36

53. Bhatt DK, Gupta S, Ploug KB, Jansen-Olesen I, Olesen J (2014) mRNA distribution of CGRP and its receptor components in the trigeminovascular system and other pain related structures in rat brain, and effect of intracerebroventricular administration of CGRP on Fos expression in the TNC. Neurosci Lett 559:99-104
54. Kresse A, Jacobowitz DM, Skofitsch G (1995) Detailed mapping of CGRP mRNA expression in the rat central nervous system: comparison with previous immunocytochemical findings. Brain Res Bull 36(3):261-274

55. Bernard JF, Alden M, Besson JM (1993) The organization of the efferent projections from the pontine parabrachial area to the amygdaloid complex: a Phaseolus vulgaris leucoagglutinin (PHA-L) study in the rat. J Comp Neurol 329(2):201-229

56. Eftekhari S, Edvinsson L (2011) Calcitonin gene-related peptide (CGRP) and its receptor components in human and rat spinal trigeminal nucleus and spinal cord at C1-level. BMC Neurosci 12:112

57. Eftekhari S, Gaspar RC, Roberts R, Chen TB, Zeng Z, Villarreal S, Edvinsson L, Salvatore CA (2016) Localization of CGRP receptor components and receptor binding sites in rhesus monkey brainstem: a detailed study using in situ hybridization, immunofluorescence, and autoradiography. J Comp Neurol 524:90-118

58. Silberstein S, Lipton R, Dodick D, Freitag F, Mathew N, Brandes J, Bigal M, Ascher S, Morein J, Wright P, Greenberg S, Hulihan J (2009) Topiramate treatment of chronic migraine: a randomized, placebo-controlled trial of quality of life and other efficacy measures. Headache 49:1153-1162

59. Diener HC, Bussone G, Van Oene JC, Lahaye M, Schwalen S, Goadsby PJ, TOPMAT-MIG-201(TOP-CHROME) Study Group (2007) Topiramate reduces headache days in chronic migraine: a randomized, double-blind, placebocontrolled study. Cephalalgia 27:814-823

60. Ambrosini A, Schoenen J (2006) Electrophysiological response patterns of primary sensory cortices in migraine. J Headache Pain 7:377-388

61. Shank RP, Gardocki JF, Streeter AJ, Maryanoff BE (2000) An overview of the preclinical aspects of topiramate: pharmacology, pharmacokinetics and mechanism of action. Epilepsia 41(Suppl 1):S3-S9

62. Angehagen M, Ben-Menachem E, Rönnbäck L, Hansson E (2003) Novel mechanisms of action of three antiepileptic drugs, vigabatrin, tiagabine, and topiramate. Neurochem Res 28(2):333-340

63. Benoliel R, Tal M, Eliav E (2006) Effects of topiramate on the chronicconstriction injury model in the rat. J Pain 7:878-883

64. Rus NN, Bocşan C, Vesa \$̧C, Coadă CA, Buzoianu AD (2013) Topiramate in nociceptive pain - experimental analgesia study. Human \& Vet Med Int J Bioflux Soc:70-76

65. Wild KD, Yagel SK, Shank RP (1997) The novel anticonvul-sant topiramate is antiallodynic in a rat model of neu-ropathic pain. Soc Neurosci Abstr 23:2358 abstr 918.10

66. Andreou AP, Goadsby PJ (2011) Topiramate in the treatment of migraine: a kainate (glutamate) receptor antagonist within the trigeminothalamic pathway. Cephalalgia 31(13):1343-1358

67. McLean MJ, Bukhari AA, Wamil AW (2000) Effects of topiramate on sodiumdependent action-potential firing by mouse spinal cord neurons in cell culture. Epilepsia 41 (Suppl 1):S21-SS4

68. Schiffer WK, Gerasimov MR, Marsteller DA, Geiger J, Barnett C, Alexoff DL, Dewey SL (2001) Topiramate selectively attenuates nicotine-induced increases in monoamine release. Synapse 42:196-198

69. Shank RP, Maryanoff BE (2008) Molecular pharmacodynamics, clinical therapeutics, and pharmacokinetics of topiramate. CNS Neurosci Ther Summer 14(2):120-142

70. Benarroch EE (2011) CGRP: sensory neuropeptide with multiple neurologic implications. Neurology 77(3):281-287

71. Bouhassira D, Le Bars D, Villanueva L (1987) Heterotopic activation of ad and $C$ fibres triggers inhibition of trigeminal and spinal convergent neurons in the rat. J Physiol 389:301-317

72. Durham PL, Niemann C, Cady R (2006) Repression of stimulated calcitonin gene-related peptide secretion by topiramate. Headache 46(8):1291-1295

73. Lau BK, Vaughan CW (2014) Descending modulation of pain: the GABA disinhibition hypothesis of analgesia. Curr Opin Neurobiol 29:159-164

74. Johannessen SI (1997) Pharmacokinetics and interaction profile of topiramate: review and comparison with other newer antiepileptic drugs. Epilepsia 38(Suppl 1):S18-S23

75. Matar KM, Tayem YI (2014) Effect of experimentally induced hepatic and renal failure on the pharmacokinetics of topiramate in rats. Biomed Res Int 2014:570910

76. Löscher W (2007) The pharmacokinetics of antiepileptic drugs in rats: consequences for maintaining effective drug levels during prolonged drug administration in rat models of epilepsy. Epilepsia 48(7):1245-1258

77. Motaghinejad M, Motevalian M, Shabab B (2016) Neuroprotective effects of various doses of topiramate against methylphenidate induced oxidative stress and inflammation in rat isolated hippocampus. Clin Exp Pharmacol Physiol 43(3):360-371 